\title{
Physiological Partial Aldosterone Resistance in Human Newborns
}

\author{
LAETITIA MARTINERIE, ERIC PUSSARD, LAURENCE FOIX-L'HÉLIAS, FRANCOIS PETIT, CLAUDINE COSSON, \\ PASCAL BOILEAU, AND MARC LOMBÈS
}

\begin{abstract}
Inserm, U693 [L.M., E.P., M.L.], Faculté de Médecine Paris-Sud [L.M., E.P., P.B., M.L.], Service de Génétique Moléculaire, Pharmacogénétique et Hormonologie [E.P.], Service de Biochimie [C.C.], and Service d'Endocrinologie et Maladies de la Reproduction [M.L.], Le Kremlin-Bicêtre F-94276, France; Service de Pédiatrie et Réanimation Néonatales [L.F.-L., P.B.] and Service de Biochimie [F.P.], Hôpital Antoine Béclère, Assistance Publique-Hôpitaux de Paris, Clamart F-92141, France
\end{abstract}

\begin{abstract}
In the neonatal period, the human kidney is characterized by an impaired ability to regulate water and sodium homeostasis, resembling partial aldosterone resistance. The aim of our study was to assess this hormonal insensitivity in newborn infants and to determine its relationship with neonatal sodium handling. We conducted a prospective study in 48 healthy newborns and their mothers. Aldosterone, renin, and electrolyte concentrations were measured in umbilical cords and in maternal plasma. Urinary aldosterone concentrations and sodium excretion were determined at urination within $24 \mathrm{~h}$ after birth. A significant difference was observed between aldosterone and renin levels in newborn infants compared with their mothers $(817 \pm 73$ versus $575 \pm 55 \mathrm{pg} / \mathrm{mL}$ and $79 \pm 10$ versus $15 \pm 2 \mathrm{pg} / \mathrm{mL}$, respectively, $p<0.001)$. This hyperactivation of the renin-angiotensin-aldosterone system was associated with hyponatremia and hyperkalemia in the newborn infants, and high urinary sodium loss, consistent with a partial aldosterone resistance at birth. Unlike plasma aldosterone, urinary aldosterone concentration was found highly correlated with plasma potassium concentrations, thus representing the best index for accurate evaluation of mineralocorticoid sensitivity. Our study represents a comprehensive characterization of the renin-aldosterone axis in newborn infants and provides evidence for physiologic partial aldosterone resistance in the neonatal period. (Pediatr Res 66: 323-328, 2009)
\end{abstract}

$\mathrm{T}$ he human kidney displays a tubular immaturity at birth, with sodium waste, responsible for a negative sodium balance, and an impaired ability to reabsorb water (1), which is aggravated under circumstances such as prematurity, pyelonephritis, and gastroenteritis (2). This inability to maintain homeostatic functions is a major problem encountered by pediatricians, most notably in preterm infants. Indeed, the challenge is to find a balance between the lethal risk of dehydration and the associated morbidity of excessive hydromineral supplementation, contributing to severe complications such as intraventricular hemorrhage and bronchopulmonary dysplasia (3). A better understanding of water and sodium regulation in the neonatal period is a prerequisite to propose new therapeutic strategies for the management of preterm infants.

Received February 24, 2009; accepted April 10, 2009

Correspondence: Marc Lombes, MD, PhD, Inserm U693, Faculté de Médecine Paris-Sud, 63, rue Gabriel Péri, 94276 Le Kremlin Bicêtre Cedex, France; e-mail: marc.lombes@u-psud.fr

Supported by funds from Inserm, University Paris-Sud 11 [Bonus Qualité Recherche BQR (M.L.)], European Section of Aldosterone Council [ESAC (M.L.)], and fellowships (L.M.) from the Centre Evian Pour l'Eau (Danone eaux France) and the Fonds d'Etudes pour la Recherche Médicale (Assistance Publique Hôpitaux de Paris), France.
Sodium reabsorption is mainly controlled by aldosterone, a steroid hormone synthesized in the zona glomerulosa of the adrenal gland, secondary to renin stimulation via angiotensin II and to potassium stimulation (4). In the distal nephron, aldosterone, by binding to its receptor, the mineralocorticoid receptor, a transcription factor (5), tightly regulates the expression and the activity of several transport proteins implicated in sodium, potassium, and water homeostasis, as the alpha subunit of the epithelial sodium channel (6), the Na-KATPase (7), and the aquaporin 2 (8). A possible role of a partial and transient tubular unresponsiveness to this hormone has been suggested to account for sodium waste in neonates $(9,10)$.

Indeed, high levels of plasma aldosterone have been reported in the neonatal period throughout the first year of life $(10-12)$, and several cases of transient pseudohypoaldosteronism have been reported in infants $<3$ mo of age with urinary infection $(13,14)$. This suggests that a preexistent partial hormonal resistance in young infants might aggravate the impaired renal aldosterone sensitivity secondary to urinary infections. This putative aldosterone resistance has been already proposed by many authors $(9,10)$ but has never been fully characterized. This is easily understandable given the difficulty to perform clinical investigations in the neonatal period, owing to ethical considerations concerning blood samples and urine collections in the newborn infant. Few studies have reported plasma aldosterone and renin levels at birth $(9,15,16)$, but no accurate and reliable reference values are available for either plasma aldosterone and renin concentrations or urinary aldosterone levels in newborns.

Herein, we report the first large prospective study evaluating aldosterone sensitivity in newborn infants. We measured aldosterone and renin levels in both maternal and umbilical cords plasma, together with electrolytes concentrations, and compared these values with neonatal urinary aldosterone levels, sodium and potassium excretion. We established neonatal reference values for plasma aldosterone and renin and most notably for urinary aldosterone concentration $\left(\mathrm{Aldo}_{\mathrm{u}}\right)$, which seems to be the best index for accurate evaluation of aldosterone sensitivity. We have thereby demonstrated aldosterone unresponsiveness at birth.

\footnotetext{
Abbreviations: $\mathbf{A l d o}_{\mathbf{u}}$, Urinary aldosterone concentration; FeNa, Fractional excretion of sodium
} 


\section{PATIENTS AND METHODS}

Patients. Forty-eight mother-neonate couples were included in the maternity ward of Antoine-Beclere Hospital, Clamart, France. Pregnant women aged 18- to 45-y-old (maternal age mean $\pm \mathrm{SEM}=31.9 \pm 0.6$ ) were eligible for this study. The gestational age was determined by a first-trimester ultrasound scan (gestation week $=39$ wk $5 \pm 1$ d). Pregnancies were uncomplicated for diabetes, hypertension, eclampsia, adrenal or pituitary insufficiency, or other chronic diseases. Singleton pregnancies were normal without any tocolytic treatment given within $15 \mathrm{~d}$ before delivery. No fluid had been administered to mothers prior vaginal delivery, which had to be spontaneous and eutocic. Full-term newborn infants (birth weight $=3421 \pm$ $69 \mathrm{~g}$ ) with no respiratory disease or sign of per-partum anoxia (Apgar score $<5$ at $5 \mathrm{~min}$ or cord $\mathrm{pH}<7.10$ or blood lactate $>6 \mathrm{mM}$ ) were subsequently enrolled. Exclusion criteria were abnormal antenatal ultrasound scan, prenatal corticosteroid treatment, and small for gestational age. Informed and written consent was obtained from all mothers. The study was conducted in accordance with the Declaration of Helsinki and after approval of the local ethical committee (CCPPRB; Comité Consultatif Pour les Personnes en Recherche Biomédicale, Hôpital Antoine-Béclère, Clamart, France).

Blood and urinary samples. Maternal blood samples were obtained during preanalgesia peridural checkup, the day of delivery. Immediately after delivery, neonatal venous blood samples were collected from umbilical cords on EDTA and heparinized tubes and immediately transferred to laboratory for centrifugation. Supernatants were stored at $-20^{\circ} \mathrm{C}$. Single-spot urinary samples were collected onto a gauze compress settled in the diaper during the first $24 \mathrm{~h}$ of life. Compresses not contaminated by meconium were stored at $-20^{\circ} \mathrm{C}$ before subsequent processing. A 24-h urinary collection was not performed for technical as well as ethical reasons.

Hormonal and biochemical analyses. Plasma aldosterone and renin concentrations as well as electrolytes, proteins, urea, and creatinine levels were measured in maternal and umbilical cord blood. Hemolyzed samples were excluded from the study. Urine samples extracted from compresses were used to determine aldosterone, electrolytes, and creatinine concentrations.

Active plasma renin was assessed using the Renin III generation RIA kit (Cisbio, Gif sur Yvette, France) with a sensitivity of $2.5 \mathrm{pg} / \mathrm{mL}$. The intraassay and interassay coefficients of variation were $4.5 \%$ and $7.4 \%$, respectively. Renin concentrations in plasma of healthy subjects (aged 20-40 y) were $8.1 \pm 3.7(n=50$; range, $3-16)$ in supine position. Plasma aldosterone concentrations were measured with a RIA (Aldo-Riact, CIS-Bio International, Gif sur Yvette, France) with a sensitivity limit of $7 \mathrm{pg} / \mathrm{mL}$. The intra-assay and interassay coefficients of variation were $6.7 \%$ and $8.4 \%$, respectively. Aldosterone was assayed in urine samples after acid hydrolysis of aldosterone 18 -glucuronide during $18 \mathrm{~h}$ at $30^{\circ} \mathrm{C}$. Measurements on random urine samples required a correction of urinary output of aldosterone for creatinine excretion. Electrolytes, bun, total proteins, and creatinine were measured with the automat Modular P 900 (Roche, France). Urinary creatinine concentrations were determined using a rate-blanked Jaffé based method (Roche Diagnostics). The fractional excretion of sodium $(\mathrm{FeNa})$ was defined as the equation $=$ (plasma creatinine $\times$ urinary sodium $) /($ plasma sodium $\times$ urinary creatinine) $\%$.

Statistical analyses. Results are expressed as mean \pm SEM. Statistical analyses were performed using a nonparametric Mann-Whitney test. Correlations between two parameters within one sample or between newborn infants and their respective mothers were obtained by Spearman regression analysis (Prism4, Graphpad Software, Inc., San Diego, CA), with significant threshold at 0.05 .

\section{RESULTS}

Forty-eight pairs were included in the study. We obtained 48 maternal and cord blood samples for hormonal dosages and 47 maternal and cord blood samples for biochemical analyses. Twenty-eight urinary samples were obtained from these 48 newborn infants and permitted to measure both aldosterone and electrolyte concentrations.

Distinct fetal and maternal compartments. We first compared electrolyte concentrations obtained from maternal blood samples and those obtained from the umbilical cords of their respective newborn. Neonatal and maternal plasma sodium concentrations were in the same range, with mean values of $132.6 \pm 0.7$ and $132.3 \pm 0.7 \mathrm{mM}$, respectively (Table 1 ), but
Table 1. Maternal and neonatal plasma concentrations of various parameters

\begin{tabular}{lccc}
\hline & Mothers & Newborn infants & $p$ \\
\hline $\mathrm{Na}+(\mathrm{mmol} / \mathrm{L})$ & $132.3 \pm 0.7$ & $132.6 \pm 0.7$ & 0.68 \\
$\mathrm{~K}+(\mathrm{mmol} / \mathrm{L})$ & $3.7 \pm 0.1$ & $5.7 \pm 0.3$ & $<0.0001$ \\
Proteins $(\mathrm{g} / \mathrm{L})$ & $63.7 \pm 0.6$ & $58.3 \pm 0.9$ & $<0.0001$ \\
Aldosterone $(\mathrm{pg} / \mathrm{mL})$ & $574.7 \pm 54.5$ & $817.1 \pm 77.9$ & $<0.05$ \\
Renin $(\mathrm{pg} / \mathrm{mL})$ & $14.9 \pm 1.5$ & $78.8 \pm 9.6$ & $<0.0001$ \\
\hline
\end{tabular}

Results are expressed as mean \pm SEM for 48 maternal and neonatal plasma samples for renin and aldosterone and 47 samples for $\mathrm{Na}^{+}, \mathrm{K}^{+}$, and proteins. Statistical analyses were performed using a nonparametric Mann-Whitney test.

surprisingly no correlation was observed between these two parameters (data not shown). This indicates that neonatal natremia is independent from maternal natremia that may be related to pregnancy hemodilution (17) or mineralocorticoid antagonism by high levels of progesterone during late gestation (18). Furthermore, plasma potassium concentrations were significantly higher in newborn infants than in their respective mothers, with mean values reaching $5.7 \pm 0.3$ versus $3.7 \pm$ $0.1, p<0.001$ (Table 1 and Fig. 1A). Once again, no correlation was found between neonatal and maternal kalemia (Fig. $1 A$ and $B$ ), even after excluding the extremely high kalemia values obtained from nonhemolyzed samples of two newborn infants. Plasma protein concentrations also significantly differed, underlining that maternal and fetal compartments are distinct and in part submitted to independent regulations (Table 1). On the other hand, neonatal plasma urea and creatinine concentrations at birth strictly reflected maternal levels, as demonstrated by the very high correlation between maternal and neonatal values (Fig. 1C), in accordance with the previous studies (19). Overall, these results suggest that although creatinine and urea freely diffuse through the placenta during pregnancy, ionic homeostasis in maternal and fetal compartments is at least in part controlled by distinct regulatory mechanisms. Moreover, our findings demonstrate that healthy newborn infants have well-tolerated physiologic hyponatremia and hyperkalemia, which are highly suggestive of a functional hypoaldosteronism.

Renal tubular immaturity in newborns. Urine samples of newborn infants were collected onto a gauze compress settled in the diaper during their first $24 \mathrm{~h}$ of life. This simple, original, and noninvasive method of urine collection was very efficient and allowed us to obtain $200 \mu \mathrm{L}$ up to $5 \mathrm{~mL}$ urinary samples from 28 neonates and enabled us to measure both urinary electrolyte concentrations and steroid hormone excretion. Mean urinary sodium $\left(\mathrm{Na}_{\mathrm{u}}\right)$, potassium $\left(\mathrm{K}_{\mathrm{u}}\right)$, and creatinine $\left(\mathrm{creat}_{\mathrm{u}}\right)$ concentrations were $40.2 \pm 3.8 \mathrm{mM}, 32.5 \pm 3.2$ $\mathrm{mM}$, and $5168 \pm 637 \mu \mathrm{M}$, respectively. Sodium waste was evaluated by the $\mathrm{FeNa}$, by the $\mathrm{Na}_{\mathrm{u}} / \mathrm{creat}_{\mathrm{u}}$ ratio and by the $\mathrm{Na}_{\mathrm{u}} / \mathrm{K}_{\mathrm{u}}$ ratio. Mean $\mathrm{FeNa}$ was $0.7 \pm 0.3 \%$, mean $\mathrm{Na}_{\mathrm{u}} /$ creat $_{\mathrm{u}}$ was $19.6 \pm 8.3$, and mean $\mathrm{Na}_{\mathrm{u}} / \mathrm{K}_{\mathrm{u}}$ was $1.99 \pm 0.46$. When $\mathrm{Na}_{\mathrm{u}} / \mathrm{K}_{\mathrm{u}}$ was compared with urinary concentration, defined by urinary creatinine concentration, a highly significant negative correlation was found (Fig. 2), suggesting a renal tubular immaturity at birth. This clearly indicates that alteration in urinary concentration ability is associated with compromised 

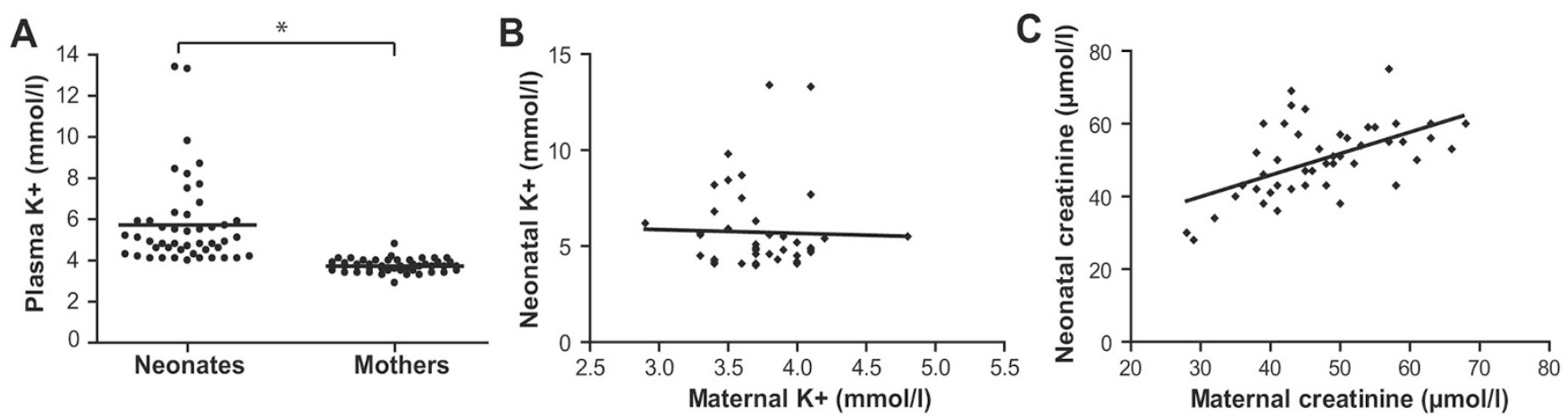

Figure 1. Comparison between maternal and neonatal electrolyte concentrations. (A) Plasma potassium concentrations in umbilical cords and maternal blood samples. Each dot represents one value and lines correspond to means of 47 values. Kalemia is significantly higher in newborn infants than in their mothers $* p<$ 0.001. (B) Correlation between maternal and neonatal kalemia. No statistical significance was reached $p=0.38$. (C) Correlation between maternal and neonatal plasma creatinine concentration; $p<10^{-4}, r=0.55$ represents the correlation coefficient. $(B, C)$ Correlations between two parameters were obtained by Spearman regression analysis.

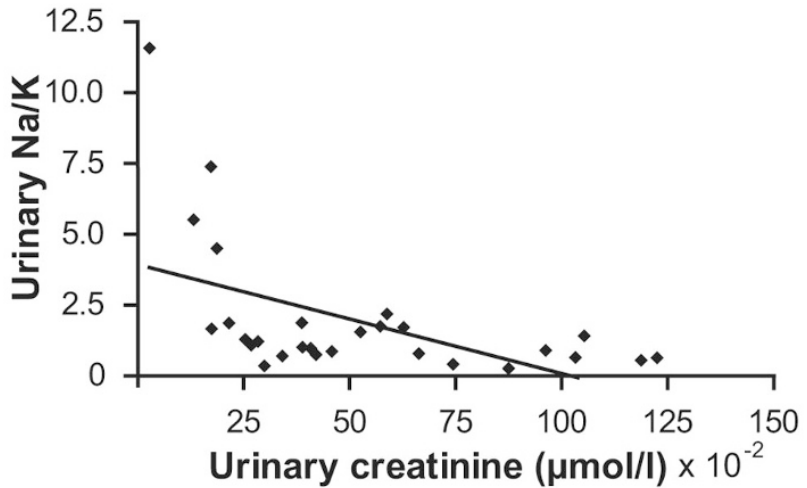

Figure 2. Neonatal renal immaturity. Correlation between urinary creatinine concentration and sodium/potassium urinary ratio $\left(\mathrm{Na}_{\mathrm{u}} / \mathrm{K}_{\mathrm{u}}\right)$ measured in 28 newborn infants. Correlation was obtained by Spearman regression analysis. $p<10^{-3}$ and correlation coefficient $r=0.61$.

sodium reabsorption, the latter being in accordance with a possible aldosterone resistance in newborn infants.

Hyperaldosteronism and hyperreninism in newborn infants. Despite biochemical signs of functional hypoaldosteronism at birth, high plasma aldosterone levels were detected in neonatal samples with mean values of $817.1 \pm 77.9 \mathrm{pg} / \mathrm{mL}$, significantly higher than those measured in maternal blood (mean values of $574.7 \pm 54.5 \mathrm{pg} / \mathrm{mL}, p<0.05$ ) (Table 1 and Fig. 3A). However, when compared with values for nonpregnant 20 - to 40 -y-old female adults $(99 \pm 43 \mathrm{pg} / \mathrm{mL}$; range, 42-201), the maternal aldosterone values were elevated, as already described during late pregnancy, associated with increased plasma progesterone levels (18). Even though neonatal and maternal aldosterone concentrations were correlated $\left(p<10^{-4}, r=0.57\right.$, data not shown) consistent with a partial transplacental passage of aldosterone from the mother to the fetus, as reported previously (20), the significant difference between neonatal and maternal mean aldosterone values provides support to a fetal neosynthesis of aldosterone. In addition, a highly significant hyperreninism was also observed in newborn infants $(78.8 \pm 9.6 \mathrm{pg} / \mathrm{mL})$ compared with maternal values $(14.9 \pm 1.5 \mathrm{pg} / \mathrm{mL}$ ) (Table 1 and Fig. $3 B$ ). Finally, as illustrated in Fig. $3 C$, the high positive correlation between neonatal plasma aldosterone and renin conclusively demonstrates that the renin-angiotensin-aldosterone system was strongly stimulated at birth.

Aldo $_{u}$ is a better index to evaluate mineralocorticoid sensitivity. From our neonatal urine samples, we were able to quantify $\mathrm{Aldo}_{\mathrm{u}}$ at birth. The Aldo $\mathrm{u}_{\mathrm{u}}$ values (mean $106.4 \pm 10.3$ $\mathrm{pg} / \mu \mathrm{g}$ of creatinine with median at $111 \mathrm{pg} / \mu \mathrm{g}$ ) followed a Gaussian distribution among 28 newborn infants (Fig.4A). Surprisingly, we did not find any correlation between plasma aldosterone concentrations and $\mathrm{Aldo}_{\mathrm{u}}$ (Fig. 4B), nor with plasma potassium concentration (Fig. $4 C$ ), suggesting that the plasma aldosterone value measured in umbilical cord may not constitute a meaningful hormonal parameter. In sharp contrast, $\mathrm{Aldo}_{\mathrm{u}}$ was negatively correlated with kalemia (Fig. 4D). Because aldosterone regulates potassium balance, $\mathrm{Aldo}_{\mathrm{u}}$, which probably reflects long-term aldosterone secretion, therefore it seems as the best index to accurately assess mineralocorticoid sensitivity during the postnatal period.

Physiologic aldosterone resistance in newborn infants. Because it is well established that aldosterone directly regulates renal sodium and potassium transport, we next examined the relationship between $\mathrm{Aldo}_{\mathrm{u}}$ and urinary electrolytes excretion in newborn infants. Interestingly, $\mathrm{Aldo}_{\mathrm{u}}$ was neither correlated with urinary potassium excretion (Fig. 5A) nor with urinary $\mathrm{Na}_{\mathrm{u}} / \mathrm{K}_{\mathrm{u}}$ ratio (Fig. $5 B$ ). This was also true for the FeNa (data not shown). These results, along with hyponatremia, hyperkalemia, and high plasma aldosterone levels, unambiguously demonstrate the physiologic partial aldosterone resistance in newborn infants. Incidentally, the newborn with the highest kalemia $(13.4 \mathrm{mM})$ presented with the highest sodium waste $(\mathrm{FeNa}=7.6 \%)$ and the most diluted urine (creat $\mathrm{u}_{\mathrm{u}} 279$ $\mu \mathrm{M})$. Collectively, our findings suggest a tight association between the severity of renal tubular immaturity and the importance of aldosterone resistance in neonates.

\section{DISCUSSION}

In this large prospective study, we demonstrated that healthy newborn infants exhibit partial aldosterone resistance with high plasma levels of aldosterone and renin, contrasting 

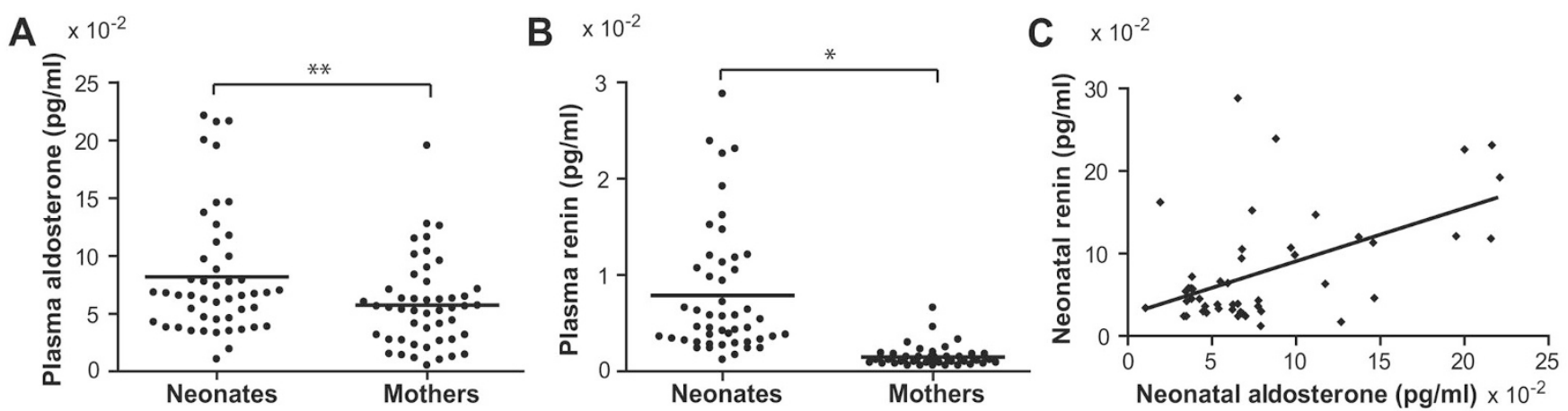

Figure 3. Hypersecretion of aldosterone and renin in newborn infants. (A) Plasma aldosterone concentrations in umbilical cords and maternal blood samples. (B) Plasma renin concentrations in umbilical cords and maternal blood samples. $(A, B)$ Each dot represents one concentration value and lines correspond to means of 48 values. ${ }^{*} p<0.01$ and $* p<0.001$. (C) Correlation between neonatal plasma aldosterone and renin concentrations. Correlation was obtained by Spearman regression analysis. $p=0.008$ and correlation coefficient $r=0.38$.
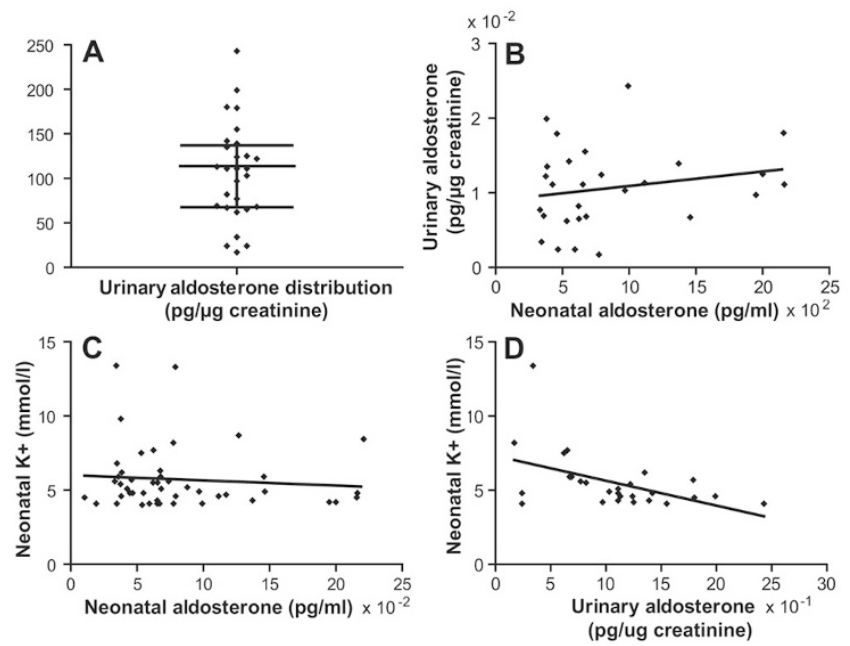

Figure 4. Aldo $_{\mathrm{u}}$ is a better index of aldosterone sensitivity at birth. (A) Distribution of $\mathrm{Aldo}_{\mathrm{u}}$ values in newborn infants. Each dot represents one value and lines correspond to median \pm interquartiles. (B) Correlation between urinary and plasma aldosterone concentrations in newborn infants. No statistical significance was reached, $p=0.34$. (C) Correlation between plasma aldosterone concentration and kalemia in newborn infants. No statistical significance was reached, $p=0.55$. (D) Correlation between $\mathrm{Aldo}_{\mathrm{u}}$ and kaliemia in newborn infants, $p=0.005$ and correlation coefficient $r=-0.52$. $(B-D)$ Correlations between two parameters were obtained by Spearman regression analysis.

with biologic signs of functional hypoaldosteronism including hyponatremia, hyperkalemia, and urinary sodium loss. These results are in accordance with previous studies that have reported high levels of aldosterone in neonates (10) compared with their mothers (21). Although aldosterone has been previously shown to cross the placental barrier from the mother to the fetus (20), highest aldosterone levels detected in the cord blood are consistent with de novo synthesis in the fetal adrenal gland, given the very early expression of the aldosterone synthase gene starting from 13 gestational weeks (22). These findings indicate an autonomous regulation of corticosteroid hormone biosynthesis in the fetus, even though the high renin levels in cord blood are likely explained by placental biosynthesis (23). Our study also highlights that ionic balances in the maternal and fetal compartments are not strictly interrelated, most notably hyperkalemia of newborn infants contrasts with
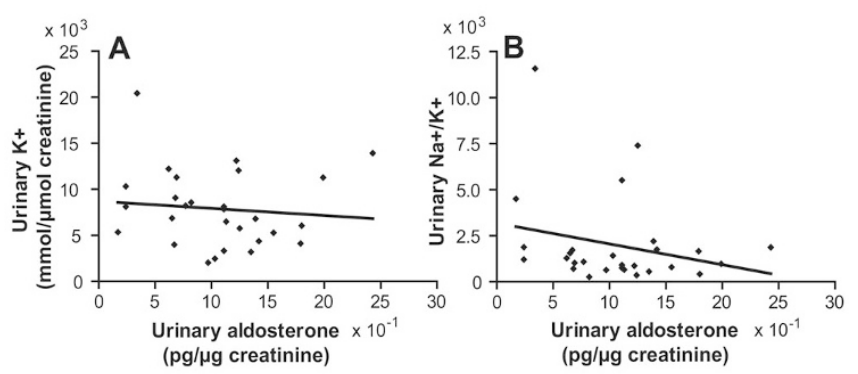

Figure 5. Aldosterone resistance in newborn infants. $(A, B)$ Correlation between urinary aldosterone and $(A)$ urinary potassium concentration, no statistical significance was reached $p=0.44$ or $(B)$ urinary sodium/potassium ratio at birth, no statistical significance was reached $p=0.28$. Correlations between two parameters were obtained by Spearman regression analysis.

maternal values, which brings new interest into the analysis of plasma electrolyte concentrations during the first $24 \mathrm{~h}$ of life, because it was assumed they strictly reflected maternal values. The demonstration of a relative neonatal mineralocorticoid insensitivity strongly suggests that it results from the inability of the fetal tubular segments to adequately respond to aldosterone action.

One of the main questions remains why and how such a physiologic hormonal unresponsiveness occurs at birth. The perfect integrity of the renin-angiotensin-aldosterone system with adaptive responses of the fetal adrenal gland sharply contrasts with the inability of fetal kidney to appropriately control sodium and water reabsorption. Whether hyperaldosteronism is the direct consequence of renal tubular immaturity, which leads to sodium loss and subsequent activation of the renin-angiotensin-aldosterone system, remains to be elucidated. Alternatively, impaired ability of nephronic segments to adequately respond to aldosterone action may constitute a protective mechanism against high plasma aldosterone levels. The neonatal weight loss related to extracellular fluid loss (24), which occurs during the first week after birth, is likely to be the consequence of this tubular insensitivity. However, the physiologic trigger and the role of this transient sodium and water negative balance in newborn infants are still unclear. Transient aldosterone unresponsiveness observed at birth may be part of an adaptive process from intrauterine aquatic life, 
where renal sodium reabsorption is dispensable, to extrauterine terrestrial life in which a strict sodium handling controlled by the kidney becomes essential. In this context, the low sodium content of the maternal breast milk (25) could contribute to delay the normalizing hydroelectrolytic homeostasis. On the other hand, a high plasma level of aldosterone could have a functional significance at birth, most notably in some aldosterone target tissues such as endothelial (26) and vascular smooth muscle cells in which high aldosterone levels may be required for rapid vasoactive responses through nongenomic effects, as described recently (27). Finally, a role of prostaglandins could be also evoked owing to the major increase in prostaglandin production in the neonatal period (28) and their inhibitory potential on hydroelectrolytic homeostasis (29).

From a pathophysiological perspective, it has been reported that plasma aldosterone levels and potassium concentration values are higher in low birth weight infants than normal weight infants (30). Similarly, preterm infants have higher aldosterone levels and hyperkalemia than full-term infants $(31,32)$. Given that low birth weight infants have greater risks of developing adult hypertension (33), high levels of plasma aldosterone and kalemia may thus serve as important biochemical parameters and represent risk factors for dysregulated renin-angiotensin-aldosterone system and early onset of a high blood pressure. It is very likely that neonatal sensitivity to aldosterone action varies with birth weight and could be a predictive factor of future chronic diseases with a possible involvement of gene developmental programming as recently described for glucocorticoid sensitivity (34). Finally, high aldosterone values gradually reach normal adult levels during the first year of life in full-term infants (11), somehow following the renal maturation process (1), and suggesting a progressive normalization of mineralocorticoid tubular responsiveness with age.

This physiologic aldosterone unresponsiveness identified in healthy full-term infants is only partial and transient with a rapid normalization of sodium balance at variance with that observed in pseudohypoaldosteronism type I patients, secondary to heterozygous inactivating mutation in the mineralocorticoid receptor gene (35), or in infants with aldosterone synthase deficiency (36). These genetically affected children have to be sodium supplemented during their first years of life because of a high risk of failure to thrive and dehydration, a condition similar to preterm infants.

Given the major physiologic impact of aldosterone action most notably during the critical neonatal period, it is essential to explore hormonal sensitivity at birth by means of precise and reliable biologic criteria. As one of the direct applications of this study, we propose that urinary aldosterone measurement is the best index for accurate evaluation of the mineralocorticoid effector mechanisms. Plasma aldosterone does not constitute a good parameter at birth in accordance with previous studies, which already reported the lack of correlation between kalemia and plasma aldosterone concentrations $(37,38)$. Instead, the highly significant negative relationship found between urinary aldosterone and plasma potassium concentrations establishes that aldosterone participates to the control of potassium balance. However, the slope of this linear regression, which reflects the hormonal sensitivity, may largely depend on the age and birth weight. We anticipate that a steeper correlation slope should be observed as the renal maturation and aldosterone responsiveness increase with age. Thus, determination of urinary aldosterone levels on a spot urinary collection and its subsequent comparison with $\mathrm{Na}_{\mathrm{u}} / \mathrm{K}_{\mathrm{u}}$ ratio or the urinary potassium excretion, characteristic of hormonal resistance, constitutes an interesting noninvasive method to investigate aldosterone sensitivity in preterm and full-term infants.

Further clinical investigations need to be conducted to evaluate aldosterone resistance and the regulation of the reninangiotensin-aldosterone system in preterm, full-term, low birth weight and normal birth weight infants, as well as in children and adults, to determine its evolution with time and to establish new therapeutic strategies, most notably for preterm neonates in whom sodium loss is a major problem.

Acknowledgment. We thank the nurses and the midwives of the Beclere maternity ward for their help during this study.

\section{REFERENCES}

1. Holtback U, Aperia AC 2003 Molecular determinants of sodium and water balance during early human development. Semin Neonatol 8:291-299

2. Herin P, Aperia A 1994 Neonatal kidney, fluids, and electrolytes. Curr Opin Pediatr 6:154-157

3. Bell EF, Acarregui MJ 2008 Restricted versus liberal water intake for preventing morbidity and mortality in preterm infants. Cochrane Database Syst Rev 1:CD000503

4. Bassett MH, White PC, Rainey WE 2004 The regulation of aldosterone synthase expression. Mol Cell Endocrinol 217:67-74

5. Viengchareun S, Le Menuet D, Martinerie L, Munier M, Pascual-Le Tallec L, Lombes M 2007 The mineralocorticoid receptor: insights into its molecular and (patho)physiological biology. Nucl Recept Signal 5:e012

6. Mick VE, Itani OA, Loftus RW, Husted RF, Schmidt TJ, Thomas CP 2001 The alpha-subunit of the epithelial sodium channel is an aldosterone-induced transcript in mammalian collecting ducts, and this transcriptional response is mediated via distinct cis-elements in the 5'-flanking region of the gene. Mol Endocrinol 15:575588

7. Derfoul A, Robertson NM, Lingrel JB, Hall DJ, Litwack G 1998 Regulation of the human $\mathrm{Na} / \mathrm{K}$-ATPase betal gene promoter by mineralocorticoid and glucocorticoid receptors. J Biol Chem 273:20702-20711

8. Hasler U, Mordasini D, Bianchi M, Vandewalle A, Feraille E, Martin PY 2003 Dual influence of aldosterone on AQP2 expression in cultured renal collecting duct principal cells. J Biol Chem 278:21639-21648

9. Sulyok E, Nemeth M, Tenyi I, Csaba I, Gyory E, Ertl T, Varga F 1979 Postnata development of renin-angiotensin-aldosterone system, RAAS, in relation to electrolyte balance in premature infants. Pediatr Res 13:817-820

10. Aperia A, Broberger O, Herin P, Zetterstrom R 1979 Sodium excretion in relation to sodium intake and aldosterone excretion in newborn pre-term and full-term infants. Acta Paediatr Scand 68:813-817

11. Mehta KP, Karnik SR, Sathe A, Pant R, Khatwani R, Bhise A 1992 Renal parameters during infancy. Indian Pediatr 29:1385-1390

12. Sippell WG, Becker H, Versmold HT, Bidlingmaier F, Knorr D 1978 Longitudinal studies of plasma aldosterone, corticosterone, deoxycorticosterone, progesterone, 17-hydroxyprogesterone, cortisol, and cortisone determined simultaneously in mother and child at birth and during the early neonatal period. I. Spontaneous delivery. J Clin Endocrinol Metab 46:971-985

13. Dolezel Z, Starha J, Novotna D, Dostalkova D 2004 Secondary pseudohypoaldosteronism in an infant with pyelonephritis. Bratisl Lek Listy 105:435-437

14. Maruyama K, Watanabe H, Onigata K 2002 Reversible secondary pseudohypoaldosteronism due to pyelonephritis. Pediatr Nephrol 17:1069-1070

15. Dillon MJ, Gillin ME, Ryness JM, de Swiet M 1976 Plasma renin activity and aldosterone concentration in the human newborn. Arch Dis Child 51:537-540

16. Fiselier T, Monnens L, van Munster P, Jansen M, Peer P, Lijnen P 1984 The renin-angiotensin-aldosterone system in infancy and childhood in basal conditions and after stimulation. Eur J Pediatr 143:18-24

17. Ekeke GI, Ebirim UA 1986 Serum sodium and potassium values in pregnant urban Nigerian and Caucasian women. Trop Geogr Med 38:28-32

18. Quinkler M, Meyer B, Bumke-Vogt C, Grossmann C, Gruber U, Oelkers W, Diederich S, Bahr V 2002 Agonistic and antagonistic properties of progesterone metabolites at the human mineralocorticoid receptor. Eur J Endocrinol 146:789-799

19. Lao TT, Loong EP, Chin RK, Lam YM 1989 Renal function in the newborn. Newborn creatinine related to birth weight, maturity, and maternal creatinine. Gynecol Obstet Invest 28:70-72 
20. Bayard F, Ances IG, Tapper AJ, Weldon VV, Kowarski A, Migeon CJ 1970 Transplacental passage and fetal secretion of aldosterone. J Clin Invest 49:13891393

21. Beitins IZ, Bayard F, Levitsky L, Ances IG, Kowarski A, Migeon CJ 1972 Plasma aldosterone concentration at delivery and during the newborn period. J Clin Invest 51:386-394

22. Coulter CL, Jaffe RB 1998 Functional maturation of the primate fetal adrenal in vivo: 3. Specific zonal localization and developmental regulation of CYP21A2 (P450c21) and CYP11B1/CYP11B2 (P450c11/aldosterone synthase) lead to integrated concept of zonal and temporal steroid biosynthesis. Endocrinology 139:5144-5150

23. Shaw KJ, Do YS, Kjos S, Anderson PW, Shinagawa T, Dubeau L, Hsueh WA 1989 Human decidua is a major source of renin. J Clin Invest 83:2085-2092

24. Modi N 2004 Management of fluid balance in the very immature neonate. Arch Dis Child Fetal Neonatal Ed 89:F108-F111

25. Baum M, Quigley R 2004 Ontogeny of renal sodium transport. Semin Perinatol 28:91-96

26. Schmidt BM, Georgens AC, Martin N, Tillmann HC, Feuring M, Christ M, Wehling M 2001 Interaction of rapid nongenomic cardiovascular aldosterone effects with the adrenergic system. J Clin Endocrinol Metab 86:761-767

27. Funder JW 2005 The nongenomic actions of aldosterone. Endocr Rev 26:313-321

28. Antonucci R, Cuzzolin L, Arceri A, Fanos V 2007 Urinary prostaglandin E2 in the newborn and infant. Prostaglandins Other Lipid Mediat 84:1-13

29. Bonilla-Felix M 2004 Development of water transport in the collecting duct. Am J Physiol Renal Physiol 287:F1093-F1101
30. Nader PJ, Procianoy RS 1996 [Hyperkalemia in very low birthweight infants: incidence and associated factors]. J Pediatr (Rio J) 72:143-150

31. Semama DS, Martin-Delgado M, Gouyon JB 2007 [Metabolism of potassium in preterm infants]. Arch Pediatr 14:249-253

32. Mildenberger E, Versmold HT 2002 Pathogenesis and therapy of non-oliguric hyperkalaemia of the premature infant. Eur J Pediatr 161:415-422

33. Barker DJ 2006 Adult consequences of fetal growth restriction. Clin Obstet Gynecol 49:270-283

34. Wyrwoll CS, Mark PJ, Waddell BJ 2007 Developmental programming of renal glucocorticoid sensitivity and the renin-angiotensin system. Hypertension 50:579584

35. Geller DS, Zhang J, Zennaro MC, Vallo-Boado A, Rodriguez-Soriano J, Furu L, Haws R, Metzger D, Botelho B, Karaviti L, Ha AM, Corey H, Janssens S, Corvol P, Lifton RP 2006 Autosomal dominant pseudohypoaldosteronism type 1: mechanisms, evidence for neonatal lethality, and phenotypic expression in adults. J Am Soc Nephrol 17:1429-1436

36. White PC 2004 Aldosterone synthase deficiency and related disorders. Mol Cell Endocrinol 217:81-87

37. Garcia del Rio C, Acuna D, Bustamante M, Soler A, Garcia-Torres L, Osorio C 1982 Increased activity of the renin-angiotensin-aldosterone system during the perinatal period. Rev Esp Fisiol 38:171-176

38. Bourchier D 2005 Plasma aldosterone levels in the 1st week of life in infants of less than 30 weeks gestation. Eur J Pediatr 164:141-145 